\title{
Adaptive Averaging for Improved SNR in Real-Time Coronary Artery MRI
}

\author{
Marshall S. Sussman*, Normand Robert, and Graham A. Wright
}

\begin{abstract}
A technique has been developed for combining a series of low signal-to-noise ratio (SNR) real-time magnetic resonance (MR) images to produce composite images with high SNR and minimal artifact in the presence of motion. The main challenge is identifying a set of real-time images with sufficiently small systematic differences to avoid introducing significant artifact into the composite image. To accomplish this task, one must: 1) identify images identical within the limits of noise; 2) detect systematic errors within such images with sufficient sensitivity. These steps are achieved by evaluating the correlation coefficient $(\mathrm{CC})$ between regions in prospective images and a template containing the anatomy of interest. Images identical within noise are selected by comparing the measured $C C$ values to the theoretical distribution expected due to noise. Sensitivity for systematic error depends on the SNR of the $C C\left(=S N R_{C C_{\max }}\right)$, which in turn depends on the noise, and the template size and structure. By varying the template size, $\mathbf{S N R}_{\mathbf{C C}_{\max }}$ may be altered. Experiments on phantoms and coronary artery images demonstrate that the $\mathbf{S N R}_{\mathbf{C C}_{\max }}$ necessary to avoid introducing significant artifact varies with the target composite SNR. The future potential of this technique is demonstrated on high-resolution ( $\sim 0.9 \mathrm{~mm}$ ), reduced field-of-view real-time coronary images.
\end{abstract}

Index Terms-Adaptive averaging, real-time MRI, reduced field-of-view, SNR, template matching.

\section{INTRODUCTION}

$\mathbf{R}$ EAL-TIME magnetic resonance (MR) can be used to visualize directly the coronary arteries in a manner that is robust in patient populations, where arrhythmias and/or breath holding difficulties often confound nonreal-time methods [1]. Additionally, unlike motion compensation methods, real-time MR is not dependent on a precise characterization of the complex coronary-diaphragm-electrocardiogram relationships [2], [3]. However, the high temporal resolution of real-time MR constrains the achievable signal-to-noise ratio (SNR) and/or spatial resolution. Recent developments in faster gradients and pulse sequence design [4]-[8], have begun to remove some constraints on the achievable spatial resolution. However, reduced SNR re-

Manuscript received September 7, 2003; revised March 25, 2004. This work was supported by the Heart and Stroke Foundation of Canada and the Canadian Institutes of Health Research. The Associate Editor responsible for coordinating the review of this paper and recommending its publication was G.-Z. Liang. Asterisk indicates corresponding author.

*M. S. Sussman is with the Department of Medical Biophysics, Sunnybrook Health Science Centre, University of Toronto, and also with the Department of Medical Imaging, University of Toronto, 585 University Ave., Room NCSB 1C-572, Toronto, ON M5G 2N2, Canada (e-mail: marshall.sussman@utoronto.ca).

N. Robert, and G. A. Wright are with the Department of Medical Biophysics, Sunnybrook Health Science Centre, University of Toronto, Toronto, ON M5G 2N2, Canada.

Digital Object Identifier 10.1109/TMI.2004.828677 mains an impediment to the production of high-quality real-time coronary images.

Following (or during) a real-time acquisition, SNR could be improved by combining $\sqrt{n}$ independent images to form a composite image. The SNR would be increased by a factor of $\sqrt{n}$ in this case. However, artifact may be introduced into the composite image if significant systematic differences exist between the component real-time images. Motion between the images would be one major source of such differences. To ensure minimal artifact in the composite image, an appropriate choice of component images is, therefore, essential.

One component image selection method proposed by Hardy et al. [6] in their "adaptive averaging" technique is template matching. A variety of template matching methods (cross-correlation, covariance, correlation coefficient [9]) were used to quantify the similarity between a template containing the structure of interest (i.e., the coronary artery) and equally sized regions in other images. Component images were selected based on maximal similarity to the template.

Using Hardy's general strategy, a component image selection algorithm using correlation coefficient template matching was introduced in a previous study by our group in the context of variable-density spiral imaging [8]. In the present study, a detailed theoretical and experimental analysis of this component image selection algorithm in the context of image combination is performed. In particular, factors affecting the quality of the component images selected by the algorithm are investigated. The utility of this analysis, and the effectiveness of the algorithm in general are then demonstrated through the application of image combination to high-resolution $(\sim 0.9 \mathrm{~mm})$, reduced field of view (FOV), real-time coronary artery images.

\section{THEORY}

The theory section is divided into three components: The first reviews the concepts behind image combination; the second describes our component image selection algorithm. Although some of the earlier material in this section has been discussed previously [8], [10], we include it here for clarity and completeness. The third section provides a detailed analysis of the the factors affecting the component image selection algorithm's ability to detect systematic errors.

\section{A. Image Combination}

A composite image $f_{\text {comp }}(x, y)$ is generated by adding $n$ component images $f_{i}(x, y)$ together

$$
f_{\text {comp }}(x, y)=\sum_{i=1}^{n} f_{i}(x, y) / n
$$


where the division by $n$ is used for normalization. If the set of component images satisfy

$$
f_{i}(x, y)=h_{0}(x, y)+\mathcal{N}\left(0, \sigma_{\text {noise }}\right) ; \quad i=1, \ldots, n
$$

where $h_{0}(x, y)$ is some underlying common structure, and $\mathcal{N}\left(0, \sigma_{\text {noise }}\right)$ represents a noise term that is white, additive and Gaussian with zero mean and standard deviation $\sigma_{\text {noise }}$, then the resulting composite image and SNR will be given, respectively, by [11]

$$
\begin{aligned}
f_{\text {comp }}(x, y) & =h_{0}(x, y)+\mathcal{N}\left(0, \sigma_{\text {noise }} / \sqrt{n}\right) \\
\operatorname{SNR}_{\text {comp }} & =\left[h_{0}(x, y) /\left(\sigma_{\text {noise }} / \sqrt{n}\right)\right] \\
& =\operatorname{SNR}_{0} \sqrt{n}
\end{aligned}
$$

where $\operatorname{SNR}_{0}\left(\equiv h_{0}(x, y) / \sigma_{\text {noise }}\right)$ is the $\mathrm{SNR}$ in the individual component images.

The major challenge in performing image combination is identifying an appropriate set of component images. Unfortunately, identifying images that are identical except for noise [i.e., (2)) is generally not possible in most practical situations. In particular, if noisy component images are selected based on a comparison to other noisy images (as in the present case), the best that can be accomplished is to identify a set of images that are identical within the limits of noise. This distinction is important because it does not preclude the possibility of noise masking the presence of small systematic differences $\left(\equiv \delta_{\text {sys }_{i}}\right)$ between the selected component images

$$
\begin{array}{r}
f_{i}(x, y)=h_{0}(x, y)+\mathcal{N}\left(0, \sigma_{\text {noise }}\right)+\delta_{\text {sys }_{i}} ; \quad i=1, \ldots, n \\
\text { where } \delta_{\text {sys }_{i}} \ll \sigma_{\text {noise. }}
\end{array}
$$

The presence of small systematic errors potentially has a deleterious effect on the resulting composite image because, unlike noise, the systematic error level is not necessarily reduced following combination [11]. To generate a composite image with improved SNR without introducing significant artifact, the component image selection algorithm must, therefore:

1) identify a set of images identical within the limits of noise;

2) detect systematic errors with sufficient sensitivity so as to avoid introducing significant artifact into the composite images.

Provided sufficient sensitivity can be achieved, image combination can increase SNR to any level.

\section{B. Identifying Component Images Identical Within the Limits of Noise}

This section reviews our method for using CC template matching to identify a set of component images with regions identical within the limits of noise.

Template matching begins with the extraction, from some initial image, of a rectangular template $h(x, y)=h_{0}(x, y)+$ $\mathcal{N}\left(0, \sigma_{\text {noise }}\right)$ of size $M \times N$ pixels containing the structure of interest (i.e., the coronary artery). The template is compared to equally sized regions at every location $(\xi, \eta)$ in a subsequent image $f(x, y)$ by calculating the correlation coefficient

$$
\begin{aligned}
\mathrm{CC}(\xi, \eta)=\sum_{i=1}^{M} \Sigma_{j=1}^{N}\left[f_{(\xi, \eta)}(x, y)\right. & \\
& \left.-\bar{f}_{(\xi, \eta)}\right][h(x, y)-\bar{h}] /\left(\sigma_{f_{(\xi, \eta)}} \sigma_{h} M \times N\right)
\end{aligned}
$$

where $f_{(\xi, \eta)}(x, y)$ is an $M \times N$ region in the image located at $(\xi, \eta)$, and $\bar{f}_{(\xi, \eta)}, \sigma_{f_{(\xi, \eta)}}$ are its mean and standard deviation (similarly for $h(x, y)$ ). At the location of the maximum CC

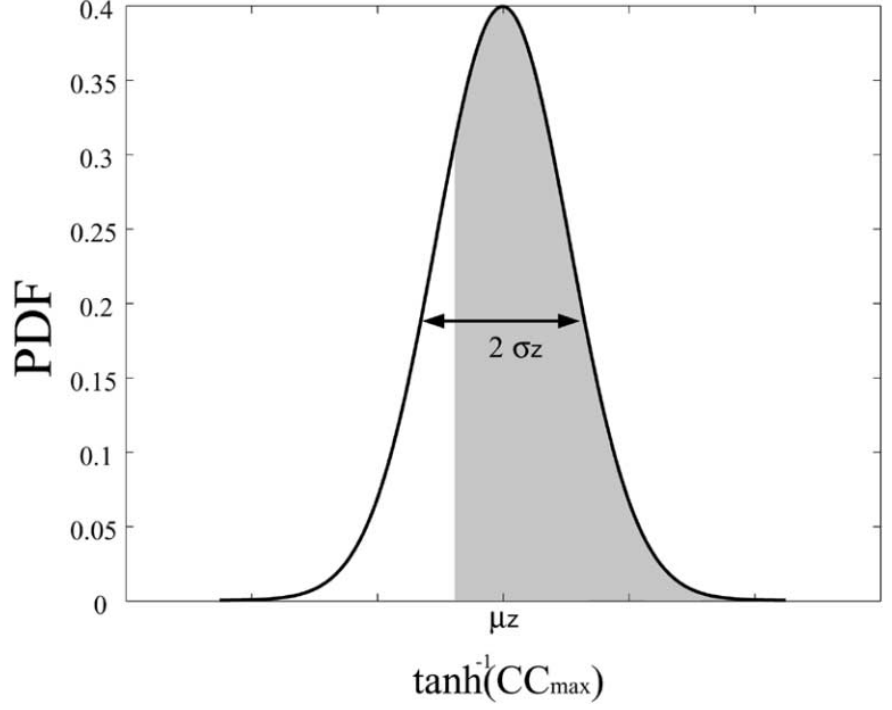

Fig. 1. Component image selection. The curve represents the Gaussian PDF of the $\mathrm{CC}_{\max }$ values under Fisher transformation. In the ideal case, the mean and standard deviation of the PDF would be given by (8), and (9), respectively. A set of component images identical within the limits of $\mathrm{CC}_{\max }$ noise would be obtained when the standard deviation of their Fisher-transformed $\mathrm{CC}_{\max }$ values equals $\sigma_{z}$. In practice, approximations inherent in the Fisher transform lead to uncertainty in the true standard deviation of the PDF. To account for this uncertainty, the modified termination condition (12) is used. However, if the true standard deviation is greater than $0.56 \sigma_{z}$, the range of $\mathrm{CC}_{\max }$ values in the selected component images would occupy only the upper range of the PDF (e.g., the shaded region under the curve). In this case, the $\mathrm{CC}_{\max }$ values would no longer be completely random samples from the distribution. Instead, they would be ordered in the sense that only the largest values in the distribution would be used.

value, the image bears the greatest similarity to the template. The maximum $\mathrm{CC}$ value itself is defined as $\mathrm{CC}_{\max }$.

To identify a set of component images identical within the limits of noise, one must analyze the $\mathrm{CC}_{\max }$ probability density function (PDF). Using Fisher's z-transform, $z=\tanh ^{-1}\left\{\mathrm{CC}_{\max }\right\}$, the $\mathrm{CC}_{\max } \mathrm{PDF}$ can be approximated by a Gaussian distribution [10], [12] with mean and standard deviation given, respectively, by

$$
\begin{aligned}
\mu_{z} & \approx \tanh ^{-1}\left\{\left\langle\mathrm{CC}_{\max }\right\rangle\right\} \\
\sigma_{z} & \approx 1 / \sqrt{M \times N-3}
\end{aligned}
$$

where the expected $\mathrm{CC}_{\max }$ value, $\left\langle\mathrm{CC}_{\max }\right\rangle$, can be calculated directly [10] as

$$
\left\langle\mathrm{CC}_{\max }\right\rangle=1-\left(\sigma_{\text {noise }} / \sigma_{h}\right)^{2} .
$$

A set of component images identical within the limits of $\mathrm{CC}_{\max }$ noise will be one whose $\mathrm{CC}_{\max }$ statistics are given by (8), (9). In practice, the component image selection algorithm uses an approach based on $\sigma_{z}$ as follows [8]: After selection of a template, an initial $n$ images are acquired, with CC template matching applied to all. The standard deviation of the Fisher-transformed $\mathrm{CC}_{\max }$ values $\left(\equiv \sigma_{\mathrm{CC}_{\max }}\right)$ is then calculated. If $\sigma_{\mathrm{CC}_{\max }}>\sigma_{z}$, data acquisition continues, with the $\mathrm{CC}$ algorithm applied to each newly acquired image. If the $\mathrm{CC}_{\max }$ value associated with the new image exceeds the smallest $\mathrm{CC}_{\max }$ value in the current $n$-image set, it replaces the image associated with that smallest $\mathrm{CC}_{\max }$ value. The $\sigma_{\mathrm{CC}_{\max }}$ value is then re-calculated on the new set. The entire procedure iterates until

$$
\sigma_{\mathrm{CC}_{\max }} \leq \sigma_{z} .
$$



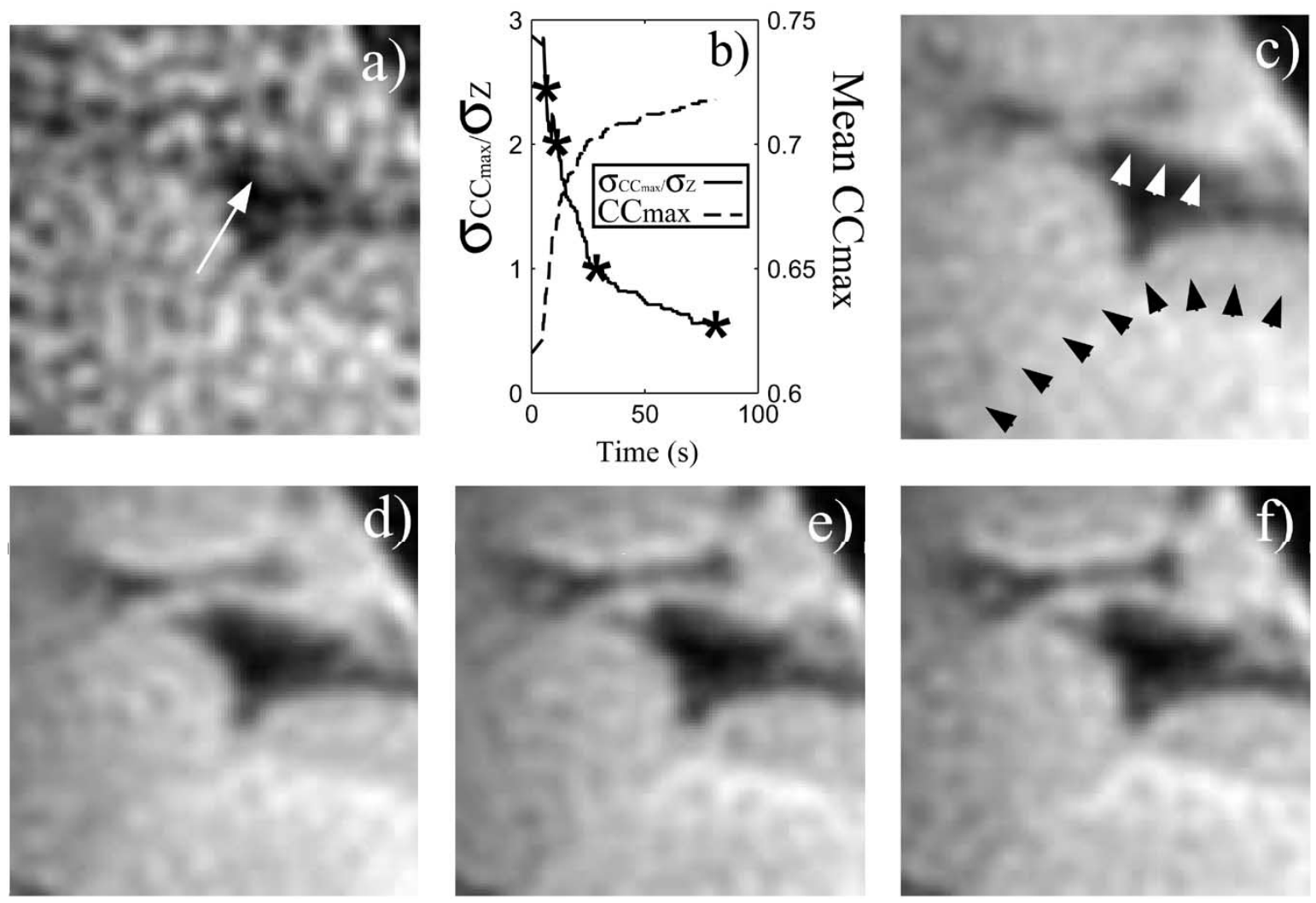

Fig. 2. Image combination $(n=25)$ of the left coronary artery (coronal-oblique view). (a) Template of size $32 \times 32$ pixels containing the left main coronary artery (white arrow) extracted from a low-SNR image. (b) Statistics of the $\mathrm{CC}_{\max }$ values of the component images as a function of time. (c)-(f) Composite images reconstructed at (c) $5.9 \mathrm{~s}\left(\sigma_{\mathrm{CC}_{\max }} / \sigma_{z}=2.5\right)$, (d) $11.2 \mathrm{~s}\left(\sigma_{\mathrm{CC}_{\max }} / \sigma_{z}=2.0\right)$, (e) $26.7 \mathrm{~s}\left(\sigma_{\mathrm{CC}_{\max }} / \sigma_{z}=1.0\right)$, and (f) the termination point at $81.1 \mathrm{~s}$. The images in (c)-(f) correspond to the points labeled by the *'s in (b). Note the increasing sharpness of the artery [white arrowheads in (c)] and the increasing border delineation [black arrowheads in (c)] in the progression from (c) to (f).

This procedure is similar to the Diminishing Variance algorithm of Sachs et al. [13], except the variance of $\mathrm{CC}_{\max }$ values rather than variance of displacement is minimized.

The component image selection algorithm requires one modification due to inadequacies of the Fisher transform approximation: In addition to template size, the standard deviation of $\mathrm{CC}_{\max }$ also has a slight dependence on the structure of the template [10]. Over different templates, the actual standard deviation was found empirically to differ from (9) by $-19.2 \% \pm$ $12.2 \%$ (mean \pm standard deviation) [10]. To account for this deviation, the algorithm termination condition is modified to the $98 \%$ confidence level $\left(=[1-19.2 \%-2 \times 12.2 \%] \sigma_{z}\right)$ for the $\mathrm{CC}_{\max }$ standard deviation

$$
\sigma_{\mathrm{CC}_{\max }} \leq 0.56 \sigma_{z}
$$

Note that (12) differs from the previous implementation of our component image selection algorithm [10], where a value of $0.7 \sigma_{z}$ was used based on a less rigorous quantitative analysis. One consequence of (12) that has not been mentioned before is that the $\mathrm{CC}_{\max }$ values of the selected component images may not be completely random samples from the $\mathrm{CC}_{\max } \mathrm{PDF}$, but may instead have an ordering (see Fig. 1). This could affect the noise distribution within the component images [14]. For example, if the true standard deviation is significantly larger than $0.56 \sigma_{z}$, there may be a correlation between both the anatomical structure and the noise in the template and component images. If significant noise correlation occurs, the SNR in the composite image may be reduced. Note that these problems could be avoided if one were to calculate the $\mathrm{CC}_{\max } \mathrm{PDF}$ numerically. However, this would have to be done separately for each individual template.

One final issue that must be addressed is that, since template matching searches every location in the two-dimensional (2-D) image for the optimal match, rigid-body displacements may exist between the selected component images. To avoid blurring in the composite image [15], these translations, which are determined from the $\mathrm{CC}_{\max }$ locations, are removed before combination.

An example image combination is displayed in Fig. 2. Early in the scan, Fig. 2(b) indicates that $\sigma_{\mathrm{CC}_{\max }} \gg \sigma_{z}$, implying significant systematic differences between the component images. A composite image generated at an early time point [Fig. 2(c)] exhibits improved SNR, but significant artifact (e.g., vessel blurring and poor border delineation). As the scan progresses, $\sigma_{\mathrm{CC}_{\max }}$ decreases, implying increased similarity among the component images. This increased similarity is reflected in improved composite image quality [Fig. 2(d), (e)]. After 81s, the termination point (12) is reached [Fig. 2(f)].

\section{Sensitivity for the Detection of Systematic Errors}

The algorithm described in the previous section does not preclude the presence of small systematic differences among the images [see (6)]. To avoid introducing significant artifact into the composite image, such differences must be sufficiently small. The ability of CC template matching to detect systematic errors between an image and template will depend on the 


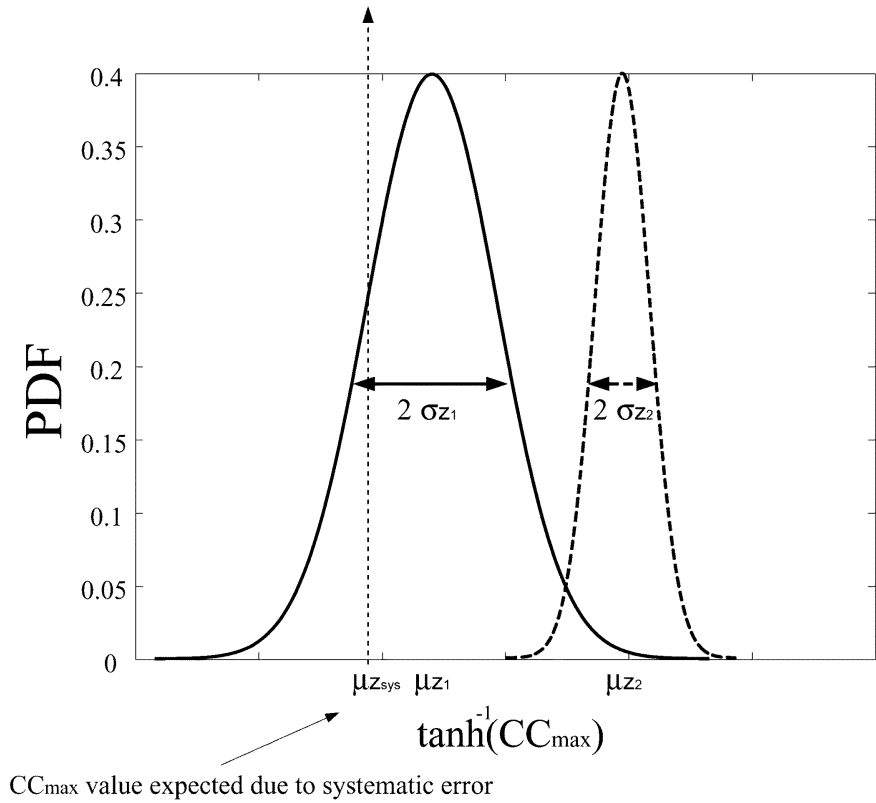

Fig. 3. The meaning of sensitivity for systematic error detection. The solid curve represents the distribution of Fisher-transformed $\mathrm{CC}_{\max }$ values expected when CC template matching is applied to an ensemble of images identical except for noise. The shape of the distribution is Gaussian, with mean $\mu_{z_{1}}$ and standard deviation $\sigma_{z_{1}}$. The thin dotted line represents an example of a Fisher-transformed $\mathrm{CC}_{\max }$ value $\left(\equiv \mu_{z_{\mathrm{sys}}}\right)$ that could result if $\mathrm{CC}$ template matching is applied to an image with a small amount of systematic error Since this value lies in the range expected due to random noise, the template associated with this particular PDF would not be sensitive for the detection of this level of systematic error. On the other hand, a template with a distribution of Fisher-transformed $\mathrm{CC}_{\max }$ values described by the dashed curve (with mean $\mu_{z_{2}}>\mu_{z_{1}}$, and standard deviation $\sigma_{z_{2}}<\sigma_{z_{1}}$ ) would be capable of detecting this level of systematic error. From this figure, it can be inferred that one possible parameter for quantifying the sensitivity for systematic error detection could be the $\mathrm{SNR}_{\mathrm{CC}_{\max }}$ value $\left(=\mu_{z} / \sigma_{z}\right)$ of the template.

$\mathrm{CC}_{\max }$ PDF. Specifically, a given level of systematic error will be detected only if its associated $\mathrm{CC}_{\max }$ value can be distinguished from the range of $\mathrm{CC}_{\max }$ values expected due to random noise (see Fig. 3). For a complete description of systematic error detection, an analysis of both the $\mathrm{CC}$ algorithm and the nature of the systematic error is necessary. However, based on the description given in Fig. 3, it is hypothesized that a simpler measure that may be used to describe the major features of sensitivity for systematic error detection is the signal-to-noise ratio (SNR) of $\mathrm{CC}_{\max }$

$$
\begin{aligned}
\mathrm{SNR}_{\mathrm{CC}_{\max }} & \equiv \mu_{z} / \sigma_{z} \\
& =\tanh ^{-1}\left\{1-\left(\sigma_{\text {noise }} / \sigma_{h}\right)^{2}\right\} \cdot \sqrt{M \times N-3}
\end{aligned}
$$

It is expected that only a template with sufficiently large $\mathrm{SNR}_{\mathrm{CC}_{\max }}$ will detect systematic errors with enough sensitivity to avoid introducing significant artifact into the composite image.

To explore the relationship between sensitivity for systematic error detection and $\mathrm{SNR}_{\mathrm{CC}_{\max }}$, a method must be developed for varying $\mathrm{SNR}_{\mathrm{CC}_{\max }}$. Equation (13) indicates that $\mathrm{SNR}_{\mathrm{CC}_{\max }}$ depends on the noise, and the template structure (through the $\sigma_{h}$ term) and size. Among these parameters, $\sigma_{\text {noise }}$ will be fixed by the nature of the real-time acquisition, and the ability to manipulate $\sigma_{h}$ (for example, by changing template location) is limited by the requirement that the template cover the anatomy of in- terest. The template size, on the other hand, could potentially be varied over a broad range. To prevent a reduction in the total anatomical area covered, a template grid [16] is used as template size is reduced [see Fig. 4(a)-(c)]. The $\mathrm{CC}_{\max }$ value of each grid element is assessed independently for each image, and the component image selection algorithm is applied to each grid element separately. Different regions of different images are then combined. In this manner, template grids potentially allow one to vary the sensitivity for systematic error detection over a broad range, without a reduction in the total anatomical coverage.

One difficulty in comparing images generated from different template sizes is that, since larger templates cover more and, therefore, different, anatomy than individual smaller templates (although the same total area is covered with the grid of smaller templates), it is not immediately obvious that one can equate directly the $\mathrm{SNR}_{\mathrm{CC}_{\max }}$ values between different template sizes. To gain insight into the circumstances under which the monotonic correspondence between sensitivity for systematic error detection and template size will hold, consider the following scenario: Let $A$ and $\boldsymbol{B}$ represent two adjacent template elements, and let $C$ be a single larger template that encompasses both $A$ and $B$ (see Fig. 5). The validity of the correspondence will depend on the relationship between the anatomy in $A$ and $B$. There will be three possible such relationships.

1) $\boldsymbol{A}$ and $\boldsymbol{B}$ are completely independent: In this case, the anatomy in $A$ will convey no information about the anatomy in $B$. There will, thus, be no increase in sensitivity for systematic error detection using the larger template $C$, even if it has a larger $\mathrm{SNR}_{\mathrm{CC}_{\max }}$ value than both $A$ and $B$.

2) $\boldsymbol{A}$ and $\boldsymbol{B}$ are completely dependent: This implies that both $A$ and $B$ convey equivalent information. Therefore, if the $\mathrm{SNR}_{\mathrm{CC}_{\max }}$ is higher in $C$ than both $A$ and $B$, greater sensitivity for systematic error detection will result by using $C$.

3) $\boldsymbol{A}$ and $\boldsymbol{B}$ are partially dependent: This scenario will fall into the middle range of the above two cases. The degree of improvement in sensitivity for systematic error detection associated with using the larger template will be determined by the degree of dependence.

The utility of varying template size to alter $\mathrm{SNR}_{\mathrm{CC}_{\max }}$ will be dictated by the relative dependence of different regions of the anatomy contained within the template. In general, dependence will be more likely if the anatomy in the different regions are connected physically. In this study, since all templates are contained within the heart, it will be assumed that a strong dependence exists. The validity of this assumption will be examined in the Discussion section. With this assumption, the sensitivity for systematic error detection (and the $\mathrm{SNR}_{\mathrm{CC}_{\max }}$ value) associated with any subregion of a template can be equated with the sensitivity for systematic error detection (and the $\mathrm{SNR}_{\mathrm{CC}_{\max }}$ value) of the template as a whole [see Fig. 5(iii) and (iv)]. This allows one to compare directly the $\mathrm{SNR}_{\mathrm{CC}_{\max }}$ values associated with different template sizes.

\section{Methods And Results}

The goal of the image combination algorithm is to generate a composite image with improved SNR, without the introduction 

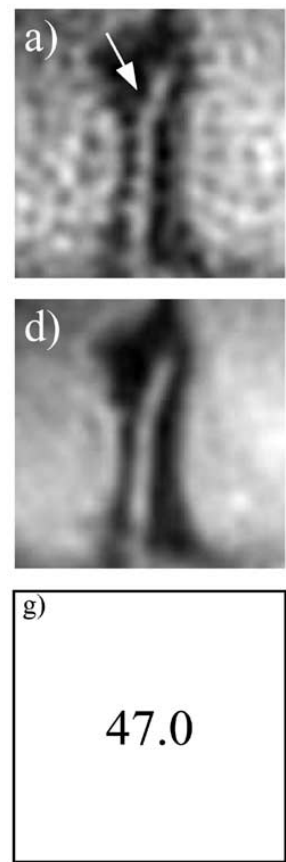
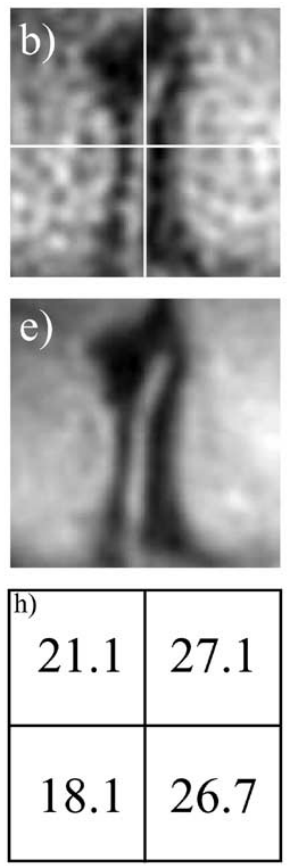
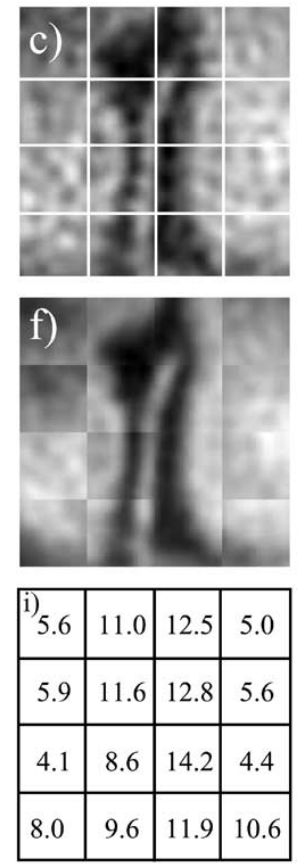

Fig. 4. Image combination on the right coronary artery using the different template sizes. Templates of size (a) $32 \times 32$ pixels, (b) $2 \times 2$ grid of $16 \times 16$ pixels, and (c) $4 \times 4$ grid of $8 \times 8$ pixels extracted from a low-SNR real-time image. The right coronary artery is indicated by the arrow in (a). (d), (e), and (f) Composite images generated using the templates in (a), (b), and (c), respectively. (g), (h), and (i) The $\mathrm{SNR}_{\mathrm{CC}_{\max }}$ values in the regions corresponding to the template elements in (a), (b), and (c), respectively. Composite image quality is poorest at the lowest $\mathrm{SNR}_{\mathrm{CC}_{\max }}$ values.

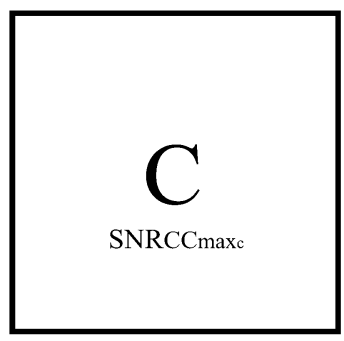

(i)

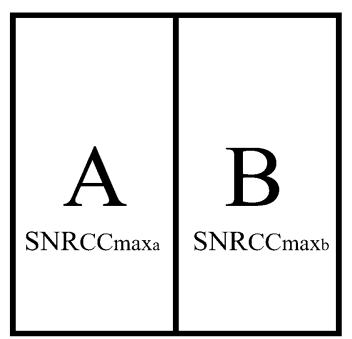

(ii)

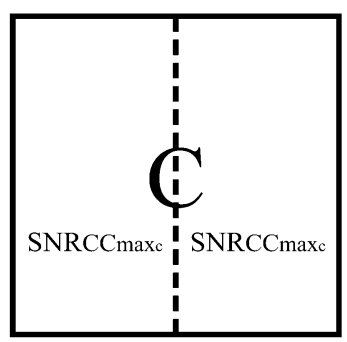

(iii)

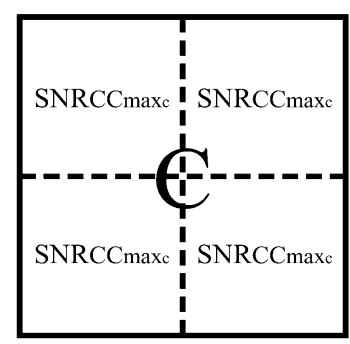

(iv)

Fig. 5. The utility of varying template size to alter the $S N R_{C C_{\max }}$ value. (i) Single and (ii) $1 \times 2$ grid template covering the same total area. Due to its larger size, template $C$ will have a larger $\mathrm{SNR}_{\mathrm{CC}_{\max }}$ value $\left(=\mathrm{SNR}_{\mathrm{CC}_{\max }}\right)$ than either $A\left(=\mathrm{SNR}_{\mathrm{CC}_{\max }}\right)$ or $B\left(=\mathrm{SNR}_{\mathrm{CC}_{\max } B}\right)$. However, if the anatomy in $A$ and $B$ are completely independent of each other (for example, if they cover entirely different anatomical structures), then the larger $S_{N R R_{C}}$ value of $C$ will not translate into a greater sensitivity for systematic error detection. On the other hand, if $A$ and $B$ are dependent, then the larger $\mathrm{SNR}_{\mathrm{CC}} \max$ value of $C$ will result in a greater sensitivity for systematic error detection. Since all templates in the present study are contained completely within the same anatomical structure (i.e., the heart), it will be assumed that the latter condition holds. Under these circumstances, when using the larger template $C$, the sensitivity for systematic error detection in any subregion will be described by $\mathrm{SNR}_{\mathrm{CC}_{\max _{C}}}$, as illustrated in the examples of (iii), (iv). This allows one to compare directly the $\mathrm{SNR}_{\mathrm{CC}} \mathrm{max}_{\mathrm{m}}$ values associated with different template sizes.

of significant artifact. To evaluate the effectiveness of the algorithm in achieving this goal, we need to 1) characterize the improvement in image quality provided by the algorithm, 2) verify that the algorithm is capable of identifying a set of component images identical within the limits of $\mathrm{CC}_{\max }$ noise in vivo, and 3 ) characterize the algorithm's sensitivity for the detection of systematic errors.

\section{A. Characterizing the Improvement in Image Quality Following Combination}

The first experiment characterizes the improvement in image quality provided by the image combination algorithm. If a set of component images could be selected randomly from an ensemble of images identical except for noise, this result would be trivial (i.e., SNR increase of $\sqrt{n}$ ). However, as discussed in the Theory section, the algorithm for selecting component images may result in an ordering of the $\mathrm{CC}_{\max }$ values, which could affect the properties of the component images. The first experiment assesses the significance of this effect by evaluating the results of image combination in the ideal case of real-time images of static phantoms. Each image was acquired using three spiral interleaves (interleaf duration $=17 \mathrm{~ms}$, in-plane resolution $=2 \mathrm{~mm}$, FOV $=20 \mathrm{~cm}$, flip angle $=30^{\circ}$, and TR $=40 \mathrm{~ms}$ ) [17]. Images were acquired continuously for a duration of $60 \mathrm{~s}$.

To evaluate the properties of the component images, the statistics of the $\mathrm{CC}_{\max }$ values (i.e., the mean and standard deviation) were compared to the values expected when $\mathrm{CC}$ template matching is applied to an ensemble of images identical 

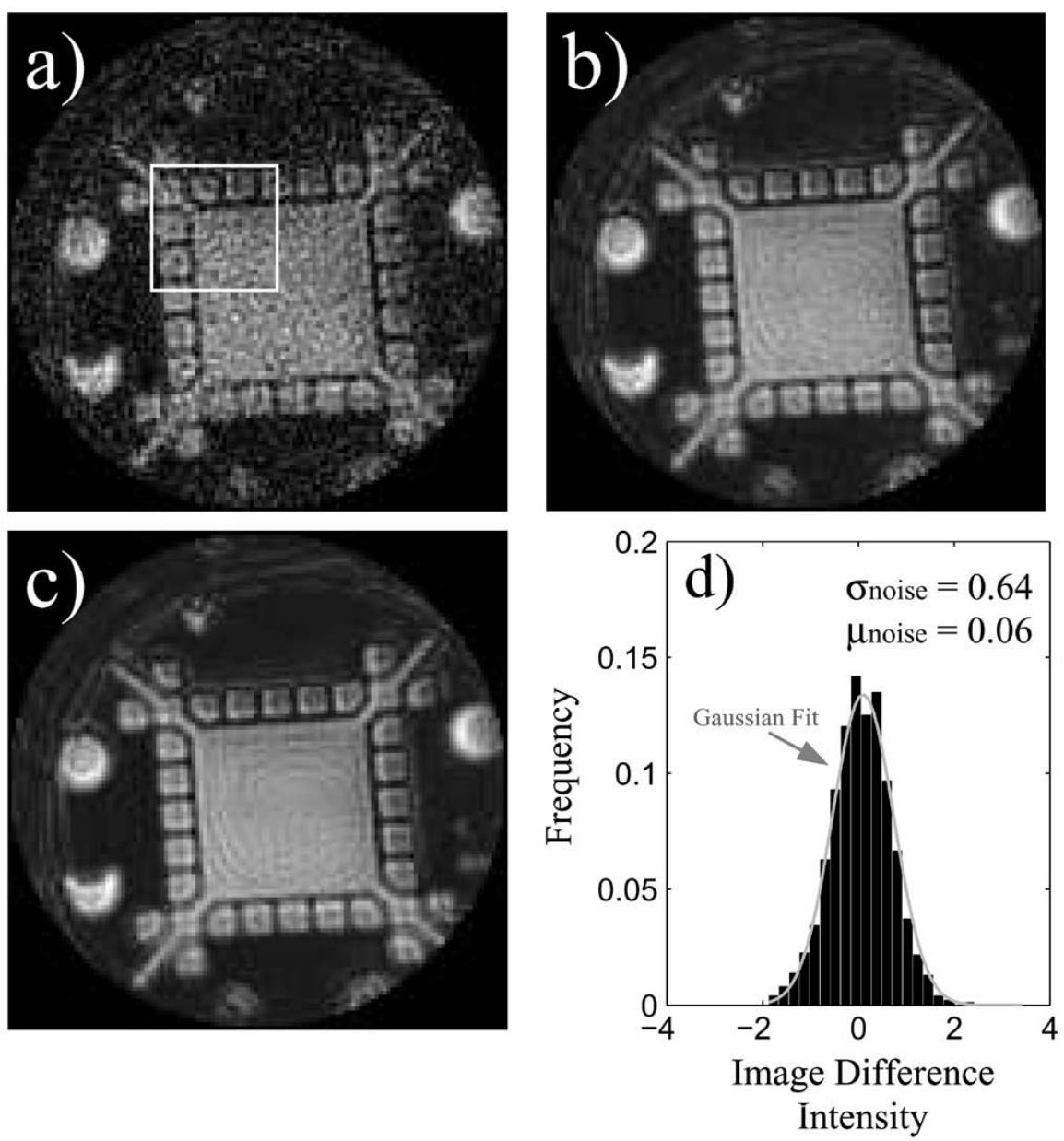

Fig. 6. Typical results of a phantom image combination. (a) Low-SNR real-time component image. The location of the template is indicated by the white rectangle. (b) High-SNR composite image $(n=25)$. (c) "Noise-free" gold standard composite image $(n=1015)$. Histogram of the template region of the difference image formed by subtracting (c) from (b). The mean $\left(\mu_{\text {noise }}\right)$ and standard deviation $\left(\sigma_{\text {noise }}\right)$ of the noise distribution were calculated from the Gaussian fit (grey curve) to the histogram.

except for noise. Since the standard deviation of the selected component images is equal by definition to (12), one need only evaluate the mean $\mathrm{CC}_{\max }$ value. This was accomplished by comparing the noise calculated from (10) ( $\left.\equiv \sigma_{\text {noisecC }}\right)$ to the true noise value $\left(\sigma_{\text {noise }}\right)$. The true noise value was determined by calculating the standard deviation of the region obtained by subtracting the template from a "noise-free" gold standard image. These latter images were obtained by adding together every image in the real-time series $(\approx 1000$ images). To evaluate the SNR and noise characteristics of the composite images, a Gaussian curve was fitted to the difference image obtained from the subtraction of the composite and "noise-free" gold standard images (see Fig. 6). To quantify the experimental uncertainty, the procedure was repeated five times using different phantoms and imaging orientations.

Results are listed in Table I. The second column indicates a significant discrepancy between $\sigma_{\text {noisecC }}$ and the true noise value. This implies a bias in the properties of the selected component images. This bias is likely due to $\mathrm{CC}_{\max }$ ordering. This hypothesis is supported by the data in the third column which indicates that, if component images are selected completely randomly from the entire series of static phantom images rather than by the algorithm, the discrepancy disappears. In terms of the composite image properties, SNR was found to increase by $\sqrt{n}(=5.11 \pm 0.16$ for 25 component images), and the noise was found to have a mean value close to zero $\left(\mu_{\text {noise }}=-0.06 \pm\right.$ $0.03)$. The shape of the noise distribution was Gaussian. Taken together, these results imply that, while the component image properties are slightly biased by $\mathrm{CC}_{\max }$ ordering, the resulting composite image properties are largely unaffected.

\section{B. Identifying Component Images In Vivo}

The success of the image combination algorithm in vivo depends on its ability to identify a set of component images identical within the limits of $\mathrm{CC}_{\max }$ noise, from within a larger pool of images that are not. To evaluate whether the algorithm is capable of accomplishing this task, the statistics of the $\mathrm{CC}_{\max }$ values must be evaluated as in the phantom experiments. However, unlike the phantom experiments, independent noise measurements could not be performed directly in the template region (since there is no noise-free image available for subtraction). Instead, independent noise measurements were made in the background regions of the template image $\left(\equiv \sigma_{\text {noise }}\right.$ ack $)$.

The real-time coronary images were acquired with the same parameters as the phantom experiments, with the exception of the use of a $10^{\circ}$, rather than a $30^{\circ}$ flip angle (this lowered the component image SNR in order to more readily demonstrate the improvement provided by image combination). Image selection was performed using templates of $32 \times 32$ pixels, $2 \times 2$ grids of $16 \times 16$ pixels, and $4 \times 4$ grids of $8 \times 8$ pixels. 
TABLE I

PROPERTIES OF THE COMPONENT IMAGES

\begin{tabular}{|c|c|c|c|c|}
\hline Template Size & $\frac{\sigma_{\text {noise }_{C C}}-\sigma_{\text {noise }}}{\sigma_{\text {noise }}}$ & $\begin{array}{c}\frac{\sigma_{\text {noise }_{C C}}-\sigma_{\text {noise }}}{\sigma_{\text {noise }}} \\
\text { (Random Selection) }\end{array}$ & $\frac{\sigma_{\text {noise }_{C C}}-\sigma_{\text {noise }} \text { back }}{\sigma_{\text {noise }}}$ & $\frac{\sigma_{\text {noise }_{C C}}-\sigma_{\text {noise }_{\text {back }}}}{\sigma_{\text {noise }_{\text {back }}}}$ \\
\hline & Phantom & Phantom & Phantom & Coronary \\
\hline $32 \times 32$ & $-0.02 \pm 0.02$ & $0 \pm 0.01$ & $-0.10 \pm 0.02$ & $-0.07 \pm 0.01$ \\
\hline $16 \times 16$ & $-0.03 \pm 0.02$ & $0 \pm 0.02$ & $-0.11 \pm 0.03$ & $-0.12 \pm 0.03$ \\
\hline $8 \times 8$ & $-0.12 \pm 0.04$ & $-0.06 \pm 0.05$ & $-0.18 \pm 0.02$ & $-0.23 \pm 0.04$ \\
\hline
\end{tabular}

Columns two through four list the data for the phantom experiments, and the last column is the coronary artery data. The second and third columns list the discrepancy between the noise calculated from the mean $\mathrm{CC}_{\max }$ values and $(10)\left(\sigma_{\text {noise }}\right)$, and the "true" noise value in the template region $\left(\sigma_{\text {noise }}\right)$ calculated by subtraction of the noise-free gold standard images (see Fig. 6). The second column used component images selected by the algorithm, and the third column used a completely random component image selection from the entire real-time series of static phantom images. The fourth column lists the discrepancy between $\sigma_{\text {noise }}$ and an independent noise measurement in the background $\left(\sigma_{\text {noise }_{\text {back }}}\right)$. The last column is the same as the fourth, but calculated on the in vivo coronary data. All data are presented as the mean \pm standard error.

Twenty-five component images were used to ensure an acceptable composite SNR $\gtrsim 20$ [18], given that the component images had $\mathrm{SNR} \approx 5-10$. The procedure was performed on eight individuals.

Results are listed in the last column of Table I. The data indicate a significant discrepancy between noise calculated from (10) $\left(\sigma_{\mathrm{CC}_{\text {noise }}}\right)$ and independent noise measurements in the background $\left(\sigma_{\text {noise }_{\text {back }}}\right)$. As in the phantom experiments, part of this discrepancy is due to $\mathrm{CC}_{\max }$ ordering (compare with the second column of Table I). The remaining discrepancy is likely due to bias in the background noise measurement. This hypothesis is supported by the fourth column of Table I, which indicates that a similar discrepancy is observed in the phantom data when $\sigma_{\text {noise }_{C C}}$ is compared to $\sigma_{\text {noise }_{\text {back }}}$, rather than the true noise value calculated in the template region. The origin of this bias has not been investigated thoroughly, but it may be due to incomplete compensation for the effects of gridding in the spiral reconstruction [19]. This could lead to different weightings in different parts of the image. As a result, the noise measured in one part of the image may not reflect the noise in another. In any event, since the effect is observed both in the ideal phantomdata and in vivo, this implies that the in vivo data behaves as expected. With the source of the observed discrepancies largely accounted for, the data indicates that the algorithm is capable of identifying a set of component images identical within the limits of $\mathrm{CC}_{\max }$ noise in vivo.

\section{Characterizing Sensitivity for Systematic Error Detection}

The third set of experiments explores the hypothesis that sensitivity for systematic error detection varies with the $\mathrm{SNR}_{\mathrm{CC}_{\max }}$ value of the template. To illustrate this relationship qualitatively, Fig. 4 displays typical results from a coronary image combination using the different template sizes. In comparison with the low-SNR templates [Fig. 4(a), (b), (c)], all composite images
[Fig. 4(d), (e), (f)] provide a significant improvement in SNR. However, the composite image generated with the $4 \times 4$ template grid [Fig. 4(f)] exhibits obvious artifacts. By observing the comparatively lower $\mathrm{SNR}_{\mathrm{CC}_{\max }}$ values associated with the $8 \times 8$ template grid elements [compare Fig. 4(g), (h), (i)], it can be seen that this result supports the hypothesis of a monotonic correspondence between systematic error detection and $\mathrm{SNR}_{\mathrm{CC}_{\max }}$. In fact, even within Fig. 4(f), local variations in image quality appear to follow local variations in $\mathrm{SNR}_{\mathrm{CC}_{\max }}$ of corresponding template grid elements. This variation is responsible for the observed "tiling" effect. Fig. 7 provides a second example of image combination. In this case, image quality is degraded in the region of low $\mathrm{SNR}_{\mathrm{CC}_{\max }}$ in the composite image generated with the $16 \times 16$ template elements [Fig. 7(e)]

The above examples provide qualitative support for the hypothesized relationship between sensitivity for systematic error detection and $\mathrm{SNR}_{\mathrm{CC}_{\max }}$. Unfortunately, an absolute quantitative characterization will generally not be possible since the specific nature of the systematic error is unknown. Instead, the strategy taken in this study is to evaluate the composite images using three different quantitative methods, and then to determine if the observed behavior is consistent with the hypothesis that systematic error detection increases with $\mathrm{SNR}_{\mathrm{CC}_{\max }}$.

1) Assessing Sensitivity for Systematic Error Detection by Comparing the Composite Images to High-SNR Gold Standards: The first two methods assess sensitivity for systematic error detection by evaluating composite image quality relative to high-SNR gold standards. Such gold-standard images (see Fig. 7(f) for an example) were obtained by performing a real-time acquisition using a $30^{\circ}$ flip angle (versus $10^{\circ}$ used to acquire the low-SNR component images). In the first method, image quality was assessed by evaluating the similarity between the composite and high-SNR gold standard images through $\mathrm{CC}$ template matching. The $\mathrm{CC}_{\max }$ value resulting from this 

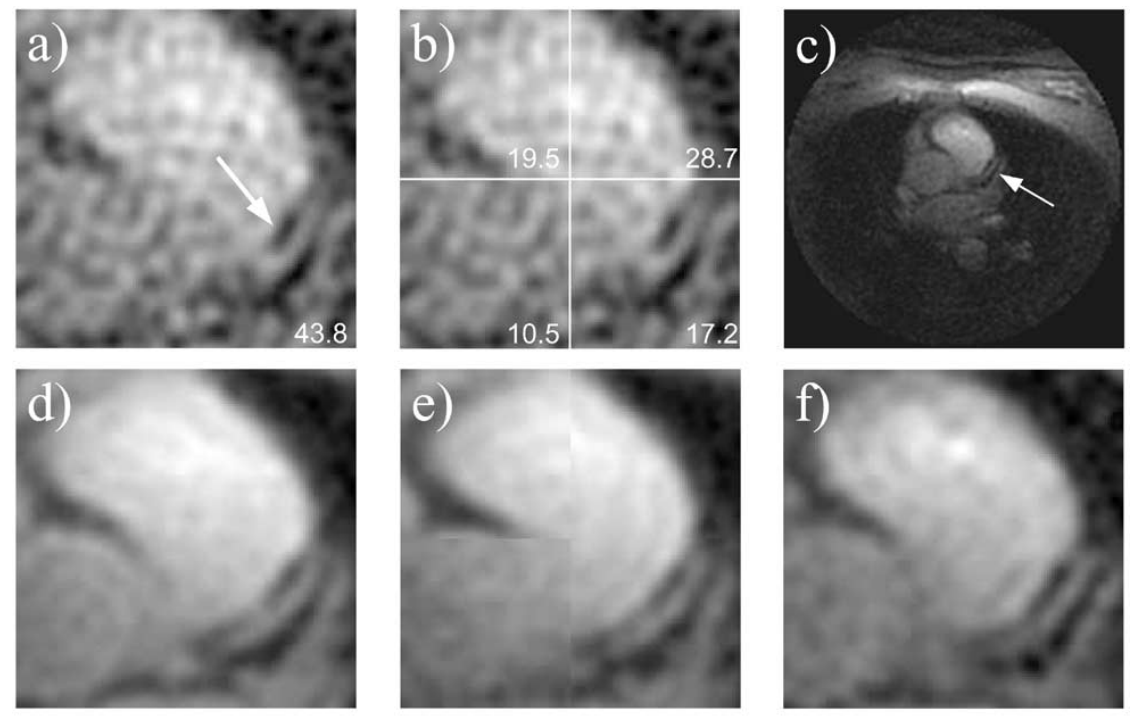

\begin{tabular}{|l|l|l|l|}
\hline g) 0.97 & 0.95 & 0.99 & 0.98 \\
\hline 0.95 & 0.97 & 0.99 & 0.99 \\
\hline 0.89 & 0.61 & 0.94 & 0.97 \\
\hline 0.82 & 0.70 & 0.73 & 0.89 \\
\hline
\end{tabular}

\begin{tabular}{|l|l|l|l|}
\hline h) -0.9 & -2.7 & 0.3 & 3.2 \\
\hline-4.9 & -2.6 & 0.3 & -1.9 \\
\hline-0.2 & -6.5 & -2.1 & -2.9 \\
\hline-1.5 & -6.4 & -6.5 & -2.8 \\
\hline
\end{tabular}

\begin{tabular}{|l|l|l|l|}
\hline i) 19.5 & 19.5 & 28.7 & 28.7 \\
\hline 19.5 & 19.5 & 28.7 & 28.7 \\
\hline 10.5 & 10.5 & 17.2 & 17.2 \\
\hline 10.5 & 10.5 & 17.2 & 17.2 \\
\hline
\end{tabular}

Fig. 7. Image combination on the left anterior descending (LAD) coronary artery using different template sizes. Templates of size (a) $32 \times 32$ pixels and (b) $2 \times 2$ grid of $16 \times 16$ pixels extracted from (c) a low-SNR image. The arrows in (a) and (c) indicate the LAD. The numbers in (a) and (b) indicate the SNR $\mathrm{CCC}_{\mathrm{max}}$ values. (d), (e) Composite images generated with the templates in (a) and (b), respectively. (f) High-SNR gold-standard image [acquired with a $30^{\circ}$ flip angle, versus $10^{\circ}$ for the low-SNR component images used to generate (d) and (e)]. (g) The $\mathrm{CC}_{\max _{\mathrm{comp}}}$ values calculated by applying CC template matching between corresponding $8 \times 8$ pixel regions in (d), (e), and (h) The discrepancy in $\mathrm{CC}_{\max _{\mathrm{comp}}}$ values from theoretical predictions $\left(=\left(\mathrm{CC}_{\max _{\mathrm{comp}}}-\left\langle\mathrm{CC}_{\max _{\mathrm{Comp}}}\right\rangle\right) /\left(\sigma_{z_{8 \times 8}}\right)\right.$. To facilitate comparison between the different values, the data in (h) is normalized by the standard deviation of $\mathrm{CC}_{\max _{\mathrm{comp}}}$ for an $8 \times 8$ pixel region $\left(=\sigma_{z} \times 8 \times 8\right.$. (i) The $\mathrm{SNR}_{\mathrm{CC}_{\max }}$ values associated with each $\mathrm{CC}_{\text {max }_{\mathrm{comp}}}$ value in (h) (compare with the values in (b), and refer to Fig. 5).

calculation ( $\left.\equiv \mathrm{CC}_{\text {max }_{\text {gold }}}\right)$ quantified the similarity. In the second method, image quality was assessed by comparing the vessel edge positions [20] in the composite and high-SNR gold standard images. If the hypothesized relationship between systematic error detection and $\mathrm{SNR}_{\mathrm{CC}_{\max }}$ is true, then one expects the similarity to increase and the discrepancy in vessel edge position to decrease with larger template $\mathrm{SNR}_{\mathrm{CC}_{\max }}$ values.

Quantitative results for the experiments are listed in Table II. The data indicate that similarity increases, and the discrepancy in vessel edge position decreases with larger template size. Note, however, that the discrepancies in vessel edge position are relatively small, implying that all templates types provide a reasonable depiction of the course of the coronary artery. By examining the $\mathrm{SNR}_{\mathrm{CC}_{\max }}$ values in the fourth column, it can be seen that these results reflect the improvement in image quality expected if sensitivity for systematic error detection varies with $\mathrm{SNR}_{\mathrm{CC}_{\max }}$.

2) Assessing Sensitivity for Systematic Error Detection Through Comparison of the Different Composite Image Types: The third method for assessing sensitivity for systematic error detection compares the similarity between composite images generated by the different template types. Specifically, composite images generated by the template grids (hereafter referred to as $f_{\mathrm{comp}_{16 \times 16}}$ and $f_{\mathrm{comp}_{8 \times 8}}$ ) are compared to the composite images generated from the $32 \times 32$ templates (hereafter referred to as $f_{\mathrm{comp}_{32 \times 32}}$ ). Similarity is evaluated by applying $\mathrm{CC}$ template matching between $f_{\mathrm{COmp}_{32 \times 32}}$ and $f_{\text {comp }_{16 \times 16}}, f_{\text {comp }_{8 \times 8}}$. To more easily elucidate changes in similarity with $\mathrm{SNR}_{\mathrm{CC}_{\max }}$, the template match is performed between every corresponding $8 \times 8$ region (i.e., the smallest template element used in the experiments) in $f_{\text {comp }_{32 \times 32}}$ and $f_{\text {comp }_{16 \times 16}}, f_{\text {comp }_{8 \times 8}}$ (see Fig. $7(\mathrm{~g}),(\mathrm{h})$, and recall that the $\mathrm{SNR}_{\mathrm{CC}_{\max }}$ value of any subregion of a template is assumed to be equal to the $\mathrm{SNR}_{\mathrm{CC}_{\max }}$ value of the template as a whole, as indicated in Fig. 5). The $\mathrm{CC}_{\max }$ value resulting from this calculation ( $\equiv C C_{\text {max }_{\text {comp }}}$ ) quantifies the similarity. If the hypothesized relationship between systematic error detection and $\mathrm{SNR}_{\mathrm{CC}_{\max }}$ is true, then the similarity between any pair of $8 \times 8$ regions in composite images will increase as the $\mathrm{SNR}_{\mathrm{CC}_{\max }}$ value associated with either region increases. However, since the $32 \times 32$ templates possess nearly uniformly larger $\mathrm{SNR}_{\mathrm{CC}_{\max }}$ values than the template grids (see Table II), the relative similarity is expected to be determined primarily by the sensitivity for systematic error detection of the template grids. Therefore, if the hypothesis is true, one expects an increase in similarity with larger template grid $\mathrm{SNR}_{\mathrm{CC}_{\max }}$ values.

Fig. 8 plots the relationship between $\left\langle\mathrm{CC}_{\max _{\text {comp }}}\right\rangle$ and $\mathrm{SNR}_{\mathrm{CC}_{\max }}$ over all of the images. To provide an absolute measure of similarity, the $\mathrm{CC}_{\max _{\text {comp }}}$ values are plotted relative to the values predicted theoretically in the case where 
TABLE II

QUANTITATIVE RESULTS OF CORONARY IMAGE COMBINATION.

\begin{tabular}{c||c|c|c}
\hline Template Size & $\tanh ^{-1}\left(C C_{\text {max }_{\text {gold }}}\right) / \sigma_{z_{8 \times 8}}$ & $\Delta$ edge (pixels) & $S N R_{C C_{\max }}$ \\
\hline \hline $32 \times 32$ & $9.4 \pm 0.4$ & $0.51 \pm 0.09$ & $39.4 \pm 7.5$ \\
\hline $16 \times 16$ & $8.5 \pm 0.4$ & $0.52 \pm 0.09$ & $17.0 \pm 6.3$ \\
\hline $8 \times 8$ & $8.3 \pm 0.4$ & $0.58 \pm 0.09$ & $7.0 \pm 2.9$ \\
\hline
\end{tabular}

The second column contains the $\mathrm{CC}_{\max }$ values calculated between the composite and high-SNR gold standard images ( $=\mathrm{CC}_{\text {max }_{\text {gold }}}$ ). The cc calculation was performed locally between every corresponding $8 \times 8$ pixel region in the composite and high-SNR gold standard images. To facilitate comparison between the different $\mathrm{CC}_{\text {max }_{\text {gold }}}$ values, the data is fisher transformed and normalized by the standard deviation of the $\mathrm{cc}_{\max }$ values $\left(=\sigma_{z_{8 \times 8}}\right)$. The third column is the absolute difference in vessel edge position ( $\equiv \Delta$ edge) between the composite and high-SNR gold standard images. The vessel tracking calculations could not be performed accurately in one of the experiments due to inadequate depiction of the vessel. In one additional experiment, vessel tracking could not be performed in the grid composite images due to poor image quality. The last column lists the $\mathrm{SNR}_{\mathrm{CC}_{\max }}$ values associated with the different template sizes. The data in the last column is listed as the mean \pm standard deviation, and the remaining columns as mean \pm standard error.

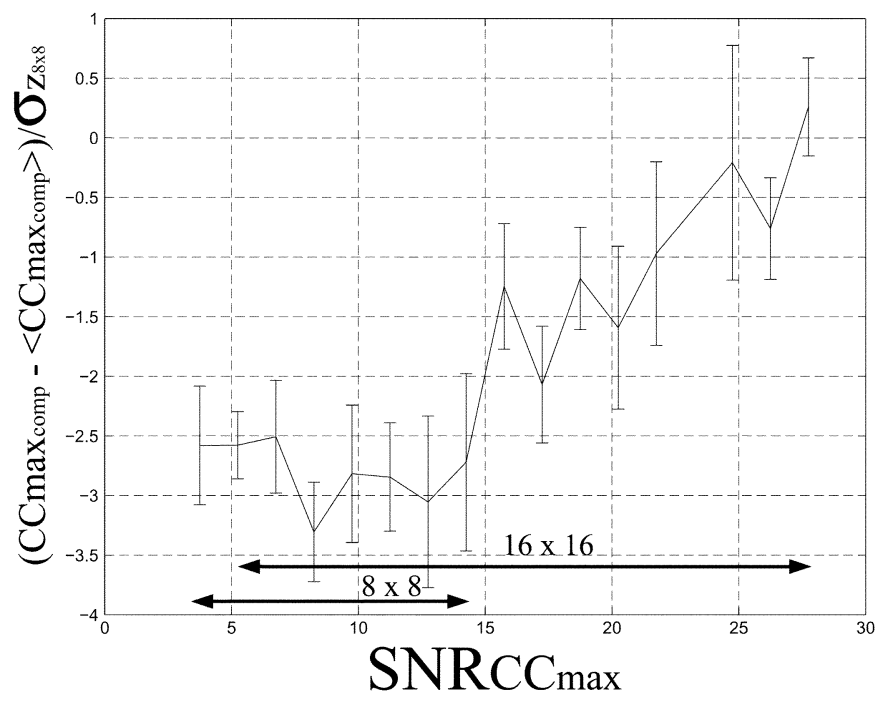

Fig. 8. Sensitivity for systematic error detection for the coronary image combination experiments. The vertical axis is the discrepancy between $\mathrm{CC}_{\max _{\text {comp }}}$ and its theoretically predicted value $\left\langle\mathrm{CC}_{\max _{\text {comp }}}\right\rangle$. To facilitate comparison between the different values, the data is normalized by the standard deviation of the $\mathrm{CC}_{\text {max }_{\text {comp }}}$ values for $8 \times 8$ templates $\left(\equiv \sigma_{z_{8 \times 8}}\right)$. The horizontal axis is the $\mathrm{SNR}_{\mathrm{CC}}$ max value in the $8 \times 8$ region of the template grid in which $\mathrm{CC}_{\text {max }}$ is calculated (see Figs. 7(h) and 5). Each point in the plot represents the mean \pm standard error over the data placed into $S N R_{\mathrm{CC}_{\max }}$ bins of width 1.5. The arrows along the bottom of the plot indicate the range of $\mathrm{SNR}_{\mathrm{CC}_{\max }}$ values associated with each grid template type (see column 4 in Table II for the mean \pm standard deviation of each template type). The decreasing discrepancy with increasing $\mathrm{SNR}_{\mathrm{CC}_{\max }}$ suggests increasing systematic error detection with increasing $\mathrm{SNR}_{\mathrm{CC}_{\max }}$.

$f_{\text {comp }_{32 \times 32}}$ and $f_{\text {comp }_{16 \times 16}}, f_{\text {comp }_{8 \times 8}}$ are identical except for noise $\left(\equiv\left\langle\mathrm{CC}_{\max _{\text {comp }}}\right\rangle\right)$. The $\left\langle\mathrm{CC}_{\max _{\text {comp }}}\right\rangle$ values were calculated from (10) and the composite noise $\left(=\sigma_{\text {noise }} / \sqrt{n}\right)$. The plot indicates a clear trend of decreasing discrepancy with increasing $\mathrm{SNR}_{\mathrm{CC}_{\max }}$. This provides further support for the hypothesis that sensitivity for systematic error detection increases with $\mathrm{SNR}_{\mathrm{CC}_{\max }}$. Note, however, that there is a fair degree of variability in the data. This is likely due to the fact that systematic error detection will also partially depend on the nature of the error, which is not accounted for by $\mathrm{SNR}_{\mathrm{CC}_{\max }}$. Additional variability may also be due to a partial breakdown of the assumption that similarity is determined solely by the $\mathrm{SNR}_{\mathrm{CC}_{\max }}$ value of the template grids, and due to violations in the assumption of complete dependence between the different regions of the template.

The plot in Fig. 8 achieves a value of zero discrepancy for $\mathrm{SNR}_{\mathrm{CC}_{\text {max }}} \approx 27$. Regions of composite images produced from template grid elements with this $\mathrm{SNR}_{\mathrm{CC}_{\max }}$ value are, therefore, identical to the corresponding regions of $f_{\mathrm{comp}_{32 \times 32}}$ within the limits of noise. This suggests that these types of templates detect systematic errors at a level below the composite image noise. If this explanation is correct, one would expect the critical $\mathrm{SNR}_{\mathrm{CC}_{\max }}$ to vary with the composite SNR. This behavior is in fact observed, as the the critical $\mathrm{SNR}_{\mathrm{CC}_{\max }}$ values for composite images with $n=4,10$ component images was found to be 17 and 22, respectively.

\section{High-Resolution Real-Time Coronary Artery Imaging}

In the last set of experiments, the potential of the techniques developed in this study was demonstrated by applying image combination to high-resolution real-time coronary artery acquisitions. While many approaches could be used to generate high-resolution real-time images, a reduced FOV technique was chosen to illustrate the concept in the present study. For simplicity, the FOV was reduced using a small 3 inch surface coil. Acquisitions consisted of six interleaf spirals with a $10 \mathrm{~cm}$ FOV. In-plane resolution was either $0.86 \mathrm{~mm}$ or $0.91 \mathrm{~mm}$. The TR was $40 \mathrm{~ms}$, for a temporal resolution of $240 \mathrm{~ms}$ (partially increased through the use of a sliding window reconstruction [17]).

Fig. 9 displays the result of a reduced FOV image combination of the left main coronary artery. In the initial real-time template image [Fig. 9(a) and (b)], the spatial resolution is high $(0.86 \mathrm{~mm})$, but the low SNR $(\sim 3)$ makes the overall image quality poor. In the composite image [Fig. 9(c)], the SNR is improved substantially. For comparison, an image of the same anatomy acquired with a standard full-FOV $(20 \mathrm{~cm})$ 

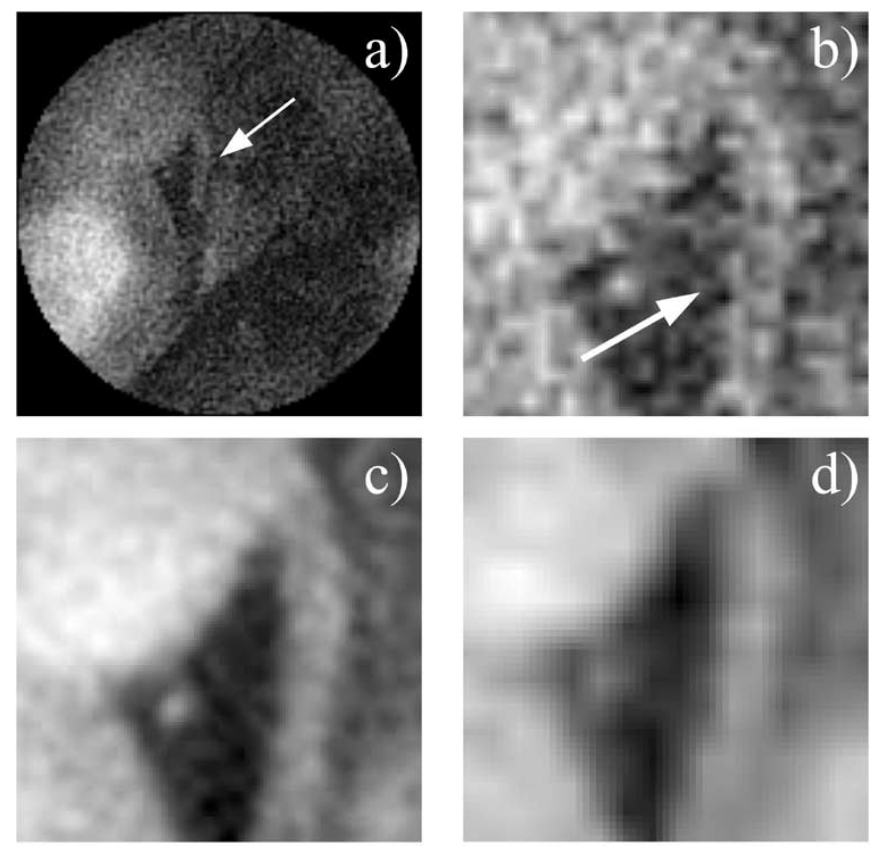

Fig. 9. Image combination of the left main coronary artery (in-plane view) on real-time component images acquired with a $10 \mathrm{~cm}$ FOV and a resolution of $0.86 \mathrm{~mm}$. (a) Low-SNR real-time image. (b) Template extracted from (a). The coronary artery is indicated by the arrows in (a) and (b). (c) Composite image $(n=36)$. (d) Image of the same anatomy acquired with a standard full FOV $(20 \mathrm{~cm})$ lower-resolution $(1.72 \mathrm{~mm})$ real-time imaging sequence.
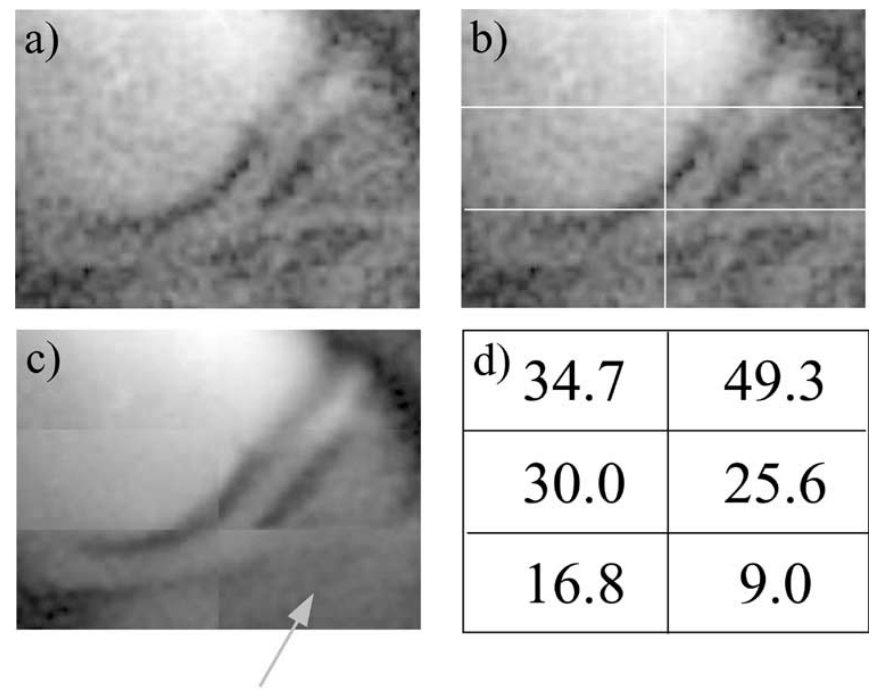

\begin{tabular}{|c|c|}
\hline d) 34.7 & 49.3 \\
\hline 30.0 & 25.6 \\
\hline 16.8 & 9.0 \\
\hline
\end{tabular}

Fig. 10. Image combination of the left coronary artery on real-time component images acquired with a $10 \mathrm{~cm}$ FOV and a resolution of $0.91 \mathrm{~mm}$. (a) Low-SNR real-time image. From this image, a $3 \times 2$ template grid of $16 \times 32$ pixels was extracted as shown in (b). (c) Composite image $(n=25)$. The region pointed out by the arrow does not provide a good depiction of the artery. (d) The $\mathrm{SNR}_{\mathrm{CC}_{\max }}$ values corresponding to each region of (b).

lower-resolution $(1.72 \mathrm{~mm})$ real-time imaging sequence is displayed in Fig. 9(d). Fig. 10 displays the results of a second reduced FOV image combination using a $3 \times 2$ grid of $16 \times 32$ templates. As with the previous example, the composite image [Fig. 10(c)] provides significantly improved image quality over the low-SNR template [Fig. 10(b)]. However, note that the portion of the coronary artery running through the region highlighted by the arrow in Fig. 10(c) is not well visualized due to the low $\mathrm{SNR}_{\mathrm{CC}_{\max }}$ in the template [Fig. 10(d)]. This result reinforces further the validity of the relationship between sensitivity for systematic error detection and $\mathrm{SNR}_{\mathrm{CC}_{\max }}$. Fig. 11 displays a final reduced FOV image combination example. The conus artery, not visible in the low-SNR template image, becomes apparent in the high-SNR composite image.

\section{DISCUSSION}

In this study, an algorithm for identifying a set of component images identical within the limits of $\mathrm{CC}_{\max }$ noise was analyzed. It was shown that the sensitivity for the detection of systematic errors of this technique depends on the $\mathrm{SNR}_{\mathrm{CC}_{\max }}$ value of the template, which in turn depends on the noise, and the template size and structure. As a rough guide, for composite coronary artery images with $\mathrm{SNR} \gtrsim 25$, a template with $\mathrm{SNR}_{\mathrm{CC}_{\max }} \gtrsim_{27}$ provided sufficient sensitivity. In this study, all of the composite images generated by $32 \times 32$ templates and some generated by $16 \times 16$ templates satisfied this criteria. However, it was also shown that this required $\mathrm{SNR}_{\mathrm{CC}_{\max }}$ varies directly with the composite SNR. Note, however, that since this study did not take into account the nature of the systematic errors in the analysis, the precise results obtained in this study may vary slightly in other situations where the nature of the systematic error may differ.

The utility of varying template size to alter $\mathrm{SNR}_{\mathrm{CC}_{\max }}$ will be dictated by the relative dependence of different regions of the anatomy contained within the template. For the analysis in this study, it was assumed that a strong dependence existed. Based on the strong relationship observed between composite image quality and $\mathrm{SNR}_{\mathrm{CC}_{\max }}$, it can be concluded that this assumption was justified. This strong dependence is likely due to the fact that all template regions were contained entirely within the heart structure itself and, thus, connected physically. This does suggest, however, that there would be likely no advantage to using a larger template that contains a mixture of anatomical structures (e.g., the heart and chest wall), since the motions of these structures would be less related.

In a previous study by our group [10], the impact of varying template size on motion tracking accuracy was explored. It was found that larger template sizes lead to greater accuracy. This result was attributed to less sensitivity to random error. This result corresponds to the observations in the present study, where larger template sizes results in less random error, thus leading to a greater capability of detecting underlying systematic errors.

To achieve as large a sensitivity for systematic error detection as possible, the above discussion implies that one should use the largest template size that contains physically connected anatomy. However, another factor to consider is the time required to identify an appropriate component image set. Fig. 12 indicates that the time required to reach the termination point generally increases with the $\mathrm{SNR}_{\mathrm{CC}_{\max }}$ value. This is likely due to the fact that the lower the tolerance for systematic error, the lower the probability of accepting a given image and, thus, the longer the time required to acquire the necessary data. Another factor which may also affect the efficiency is the nature of the anatomical motion. With the current implementation of the $\mathrm{CC}$ algorithm, images will be acceptable only if they differ by (at most) a rigid-body translation. Efficiency may be reduced using larger template sizes since rigid-body translations will be less likely over this region. To provide sufficient sensitivity 

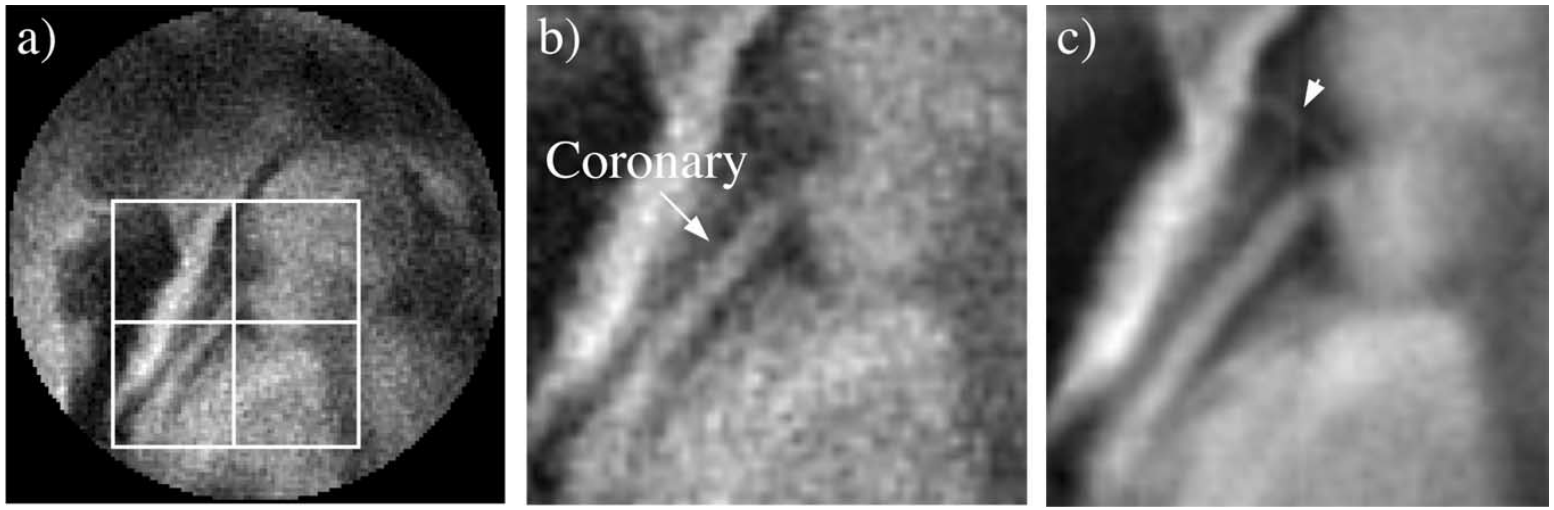

Fig. 11. Image combination of the right coronary artery on real-time component images acquired with a $10 \mathrm{~cm}$ FOV and a resolution of $0.91 \mathrm{~mm}$. (a) Low-SNR real-time image with the $2 \times 2$ grid of $32 \times 32$ templates indicated. (b) The template region extracted from (a). (c) Composite image $(n=19)$. The arrowhead indicates the conus branch, which is not apparent in the low-SNR template image (b).

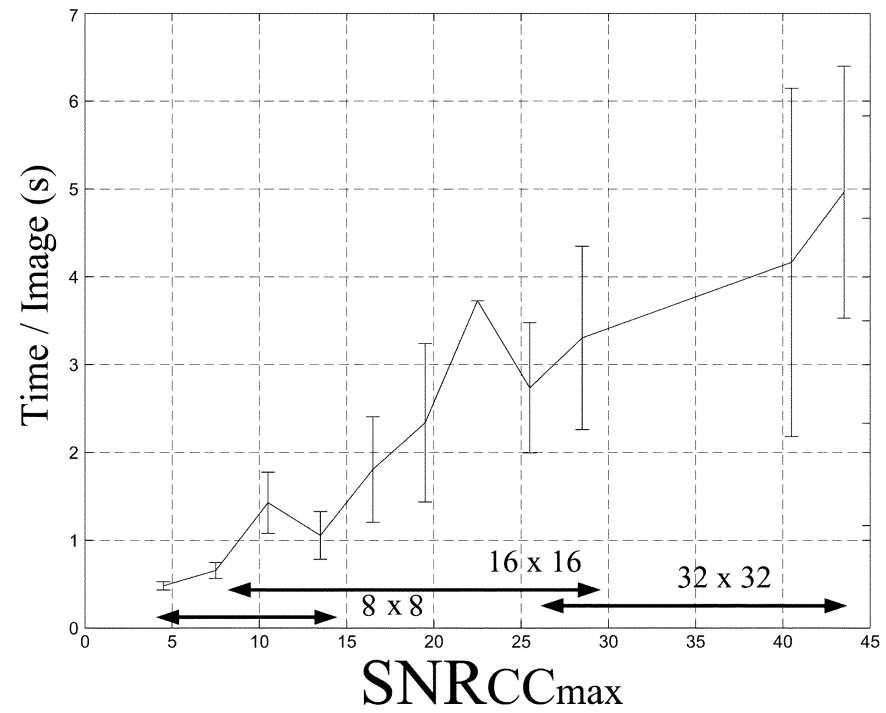

Fig. 12. Efficiency of component image selection for the coronary artery image combination experiments. The time required per component image to reach the termination point (12) is plotted against the $\mathrm{SNR}_{\mathrm{CC} \max }$ value of the templates (calculated as described in Fig. 8). Each point in the plot represent the mean \pm standard error of the data placed into $S N R_{\mathrm{CC}_{\max }}$ bins of width 3. The arrows along the bottom of the plot indicate the range of $\mathrm{SNR}_{\mathrm{CC}_{\max }}$ values associated with each template type.

for systematic error detection in the most efficient manner, the best strategy is, therefore, to use a template with an $\mathrm{SNR}_{\mathrm{CC}_{\max }}$ close to the critical value. To cover large anatomic areas in this manner may in practice require an array of different-sized templates over different regions of the anatomy, rather than a grid of equally sized templates used (for analysis purposes) in the present study.

For templates at the critical $\mathrm{SNR}_{\mathrm{CC}_{\max }}$ value, the time required to reach the termination point was about $2-3$ s/image. Since the temporal resolution of the real-time images was 120 $\mathrm{ms}$, this implies an efficiency of about $4 \%-6 \%$. This is close to the range for conventional navigator echo techniques, where efficiencies (product of navigator efficiency times cardiac window) are typically in the range of 5\%-10\% [21]. However, a direct comparison between the efficiency of conventional and image combination techniques may not be meaningful, since the termination conditions are quite different [i.e., minimal diaphragm displacement versus (12)]. There is also significant potential for improving the efficiency of image combination. For example, the termination point was set conservatively at the $98 \%$ confidence level for $\sigma_{\mathrm{CC}_{\max }}$. However, composite image quality appeared to improve quite gradually on the approach to the termination condition (e.g., see Fig. 2). With a less stringent termination condition, it may be possible to achieve significant improvements in efficiency, without a significant reduction in image quality.

In the future, this approach could be extended into three dimensions by either applying the same sorts of techniques to three-dimensional real-time images, or by applying the 2-D techniques to sequential slices.

\section{CONCLUSION}

High-quality coronary images were generated by combining a series of lower-quality real-time images. The main challenge addressed was the identification of an appropriate set of component images where the introduction of significant artifact into the composite image is avoided. A set of component images identical within the limits of noise was selected using CC template matching. It was shown that the sensitivity for the detection of systematic errors varied monotonically with the $\mathrm{SNR}_{\mathrm{CC}_{\max }}$ value of the template, which in turn increased with template size (among other factors). The $\mathrm{SNR}_{\mathrm{CC}_{\max }}$ value providing sufficient sensitivity was shown to be related to the composite SNR. After validation of the technique on standard, real-time images, its potential was illustrated through application to high-resolution, reduced FOV, real-time images of coronary arteries. As the limits of real-time imaging continue to be extended, image combination, therefore, has the potential to provide an accurate, clinically applicable method for generating high-quality images in the presence of motion.

\section{REFERENCES}

[1] A. M. Taylor, J. Keegan, P. Jhooti, P. D. Gatehouse, D. N. Firmin, and D. J. Pennell, "Differences between normal subjects and patients with coronary artery disease for three different MR coronary angiography respiratory suppression techniques," J. Magn. Reson. Imag., vol. 9, pp. 786-793, 1999.

[2] D. Manke, K. Nehrke, P. Bornert, P. Rosch, and O. Dossel, "Respiratory motion in coronary magnetic resonance angiogprahy: A comparison of different motion models," J. Magn. Reson. Imag., vol. 15, pp. 661-671, 2002 . 
[3] B. Lu, S. Mao, N. Zhuang, H. Bakhsheshi, H. Yamamoto, J. Takasu, S K. Liu, and M. J. Budoff, "Coronary artery motion during the cardiac cycle and optimal ECG triggering for coronary artery imaging," Investiga. Radiol., vol. 36, pp. 250-256, 2001.

[4] D. K. Sodickson and W. J. Manning, "Simultaneous acquisition of spatial harmonics (SMASH): Fast imaging with radiofrequency coil arrays," Magn. Reson. Med., vol. 38, pp. 591-603, 1997.

[5] K. P. Pruessmann, M. Weiger, M. B. Scheidegger, and P. Boesiger, "SENSE: Sensitivity encoding for fast MRI," Magn. Reson. Med., vol. 42, pp. 952-962, 1999.

[6] C. J. Hardy, M. Saranathan, Y. Zhu, and R. D. Darrow, "Coronary angiography by real-time MRI with adaptive averaging," Magn. Reson. Med., vol. 44, pp. 940-946, 2000.

[7] K. S. Nayak, J. M. Pauly, P. C. Yang, B. S. Hu, C. H. Meyer, and D. G. Nishimura, "Real-time interactive coronary MRA," Magn. Reson. Med., vol. 46, pp. 430-435, 2001.

[8] M. S. Sussman, J. A. Stainsby, N. Robert, N. Merchant, and G. A. Wright, "Variable-density adaptive imaging for high-resolution coronary artery MRI," Magn. Reson. Med., vol. 48, pp. 753-764, 2002.

[9] M. S. Sussman and G. A. Wright, "The correlation coefficient technique for pattern matching," in Proc. 7th Int. Conf. Soc. Magnetic Resonance in Medicine, 1999, p. 2003.

[10] — - "Factors affecting the correlation coefficient template matching algorithm with application to real-time 2-D coronary artery MR imaging," IEEE Trans. Med. Imag., vol. 22, pp. 206-216, Feb. 2003.

[11] J. R. Taylor, An Introduction to Error Analysis. Sausalito, CA: Univ. Sci. Books, 1982.

[12] R. von Mises, Mathematical Theory of Probability and Statistics. New York: Academic, 1964.
[13] T. S. Sachs, C. H. Meyer, P. Irarrazabal, B. S. Hu, D. G. Nishimura and A. Macovski, "The diminishing variance algorithm for real-time reduction of motion artifacts in MRI," Magn. Reson. Med., vol. 34, pp. 412-422, 1995.

[14] R. J. Larsen and M. M. Marx, An Introduction to Mathematical Statistics and Its Applications. Englewood Cliffs, NJ: Prentice-Hall, 1986.

[15] M. L. Wood and R. M. Henkelman, "Suppression of respiratory motion artifacts in magnetic resonance imaging," Med. Phys., vol. 13, pp. 794-805, 1986.

[16] C. Stiller and J. Konrad, "Estimating motion in image sequences," IEEE Signal Processing Mag., pp. 70-91, July 1999.

[17] A. B. Kerr, J. M. Pauly, B. S. Hu, K. C. Li, C. J. Hardy, C. H. Meyer, A. Macovski, and D. G. Nishimura, "Real-time interactive MRI on a conventional scanner," Magn. Reson. Med., vol. 38, pp. 355-367, 1997.

[18] R. S. Owen and F. W. Wehrli, "Predictability of SNR and reader preference in clinical MR imaging," Magn. Reson. Med., vol. 8, pp. 737-745, 1990.

[19] J. I. Jackson, C. H. Meyer, D. G. Nishimura, and A. Macovski, "Selection of a convolution function for fourier inversion using gridding," IEEE Trans. Med. Imag., vol. 10, pp. 473-478, Sept. 1991.

[20] N. Robert, M. J. Yaffe, and A. Langer, "Description and validation of a new quantitative coronary an-giography system," presented at the Annu. Meeting Canadian Organization of Medical Physicists and the Canadian College of Physicists in Medicine, Toronto, ON, Canada, 1994.

[21] R. M. Botnar, W. Y. Kim, P. Bornert, M. Stuber, E. Spuentrup, and W. J. Manning, "3D coronary vessel wall imaging utilizing a local inversion technique with spiral image acquisition," Magn. Reson. Med., vol. 46, pp. $848-854,2001$. 